\title{
An introduction to nanotechnology and its implications
}

\author{
Shree Kanta Subedi \\ Department of physics, Prithivi Narayan campus, Pokhara \\ Subedi.srikant@gmail.com
}

\begin{abstract}
Nanotechnology is a broad term that covers many areas of science, research and technology. In its most basic form, it can be described as working with things that are small. The area of investigation is defined by the study, design, manipulation, manufacture, and control of materials or devices by physical or chemical means at resolutions on the order of one billionth of a meter. The potential for a wide range of applications makes a basic understanding of nanotechnology important to us. This article presents an introduction to nanotechnology and discusses the implications of it that could impact the human life in the near future.
\end{abstract}

Keywords: nanoscale, quantum size effect, fullerene, buckyballs, qubits, quantum computers

\section{INTRODUCTION}

Nanotechnology involves research and technology development at the atomic, molecular or macromolecular levels in the range of approximately 1-100 nanometers to provide fundamental understanding of phenomena and materials at the nanoscale. The nanometer scale is about a billionth of a meter. In comparision, a human hair is about 10,000 nanometers in diameter. Basically nanotechnology is used to create structures, devices and systems that have novel properties and functions because of their minute size. Actually, the matter shows unusual physical and chemical properties due to increase in surface area compared to volume as particles get smaller in size \& this is called quantum size effect. This means the bulk properties of materials at nanoscale can be very different from those at larger scale. Taking the advantage of these characteristic of material, scientist designs and produces devices by manipulating the shape and size at nano scale with wide-range of implications which could include medicine, electronics, military applications, computing, space science and many more.

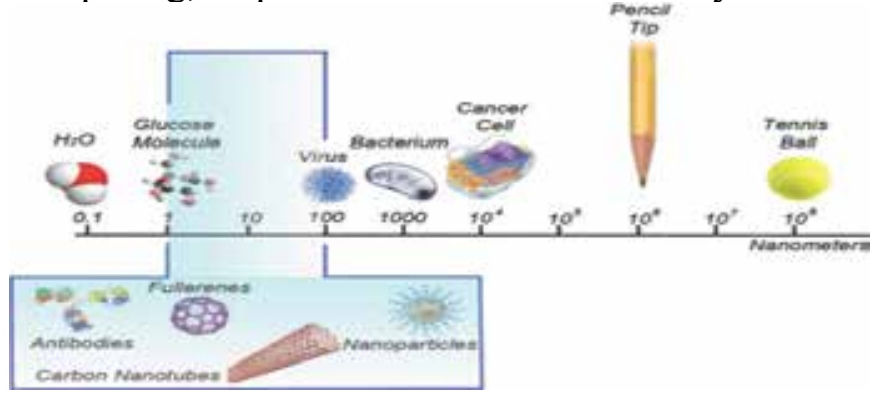

\section{HISTORICAL BACKGROUND}

The concept of nanotechnology first came from a talk given by physicist Richard Feynman entitled "There's Plenty of Room at the Bottom," at an American Physical Society meeting at Caltech on December 29, 1959 who imagined the entire Encyclopedia Britanica could be written in the head of a pin. The term "nanotechnology" was defined by Tokyo Science University Professor Norio Taniguchi in a 1974 paper as follows: "Nanotechnology' mainly consists of the processing of, separation, consolidation, and deformation of materials by one atom or by one molecule." Although scientists have been working with nanoparticles for decades, the effectiveness of their work has been circumscribed by their inability to see the structure of nanoparticles. However, after the development of scanning electron microscope in 1980 s, nanotechnology really started to take off. In 1985, when chemists discovered a football shaped molecule called buckminsterfullerene made up of 60 carbon atoms (also called fullerene or Buckyball), millions of dollars and countless man hours have been spent learning about and refining the ability to use nanotechnology. Actually a fullerene is any molecule composed entirely of carbon, in the form of a hollow sphere, ellipsoid, or tube. Spherical fullerene is called Buckyball and cylindrical one called carbon nanotube.

Fig. 1 : scale of different things 

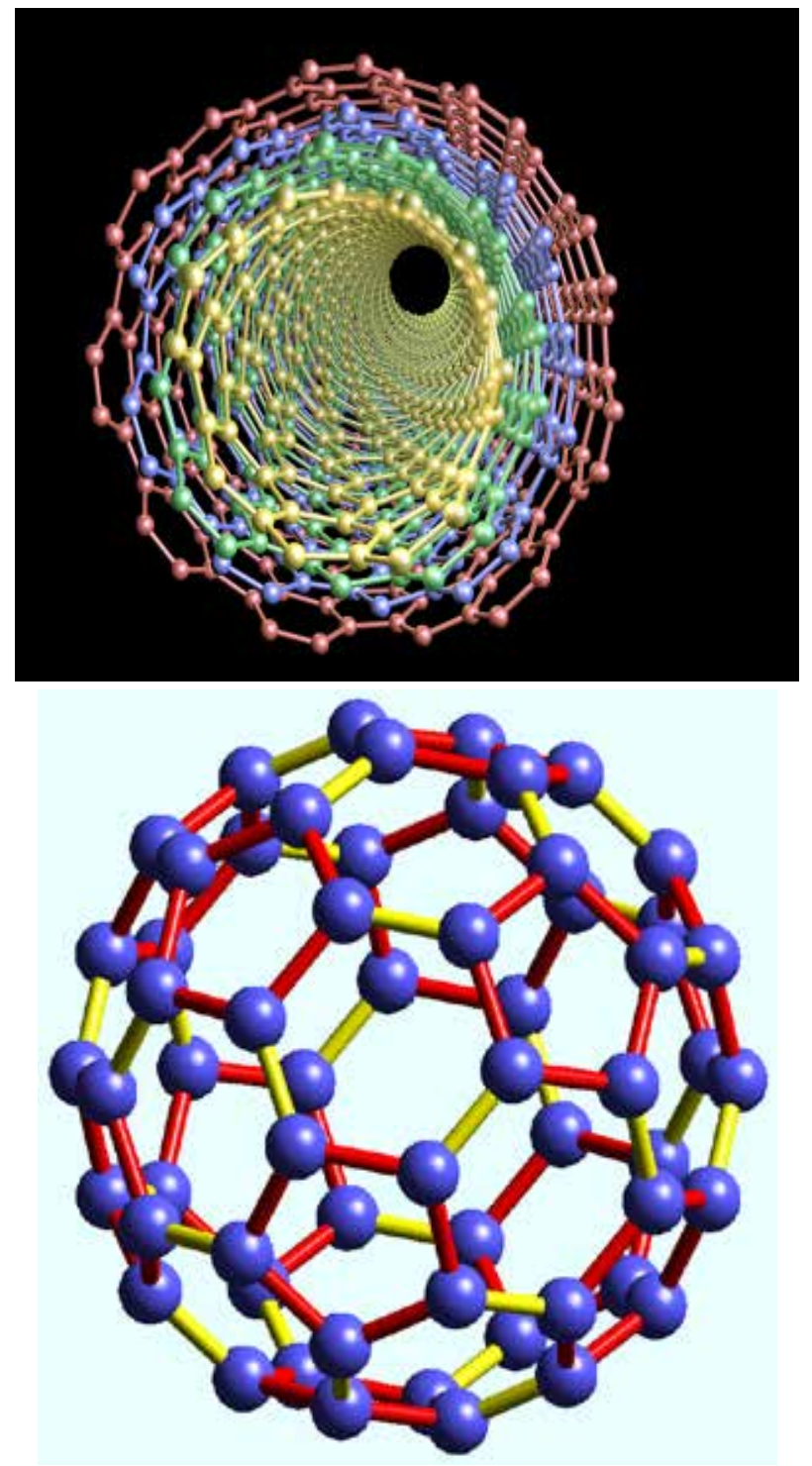

Fig 2 : carbon nanotube

Fig 3: buckyball

\section{APPLICATIONS}

While nanotechnology is seen as the way of the future and is a technology that a lot of people think will bring a lot of benefit for all who will be using it, nothing is ever perfect and there will always be pros and cons to everything. Some of them were summarised below.

\subsection{Medicine \& drug delivery}

1. With medical nanotechnology, treatment would bemore efficient and precise. Instead of opening the whole body area for surgical purposes, a microscopic nanotool would spare the patient from bloody and risky surgical process. With nanotechnology in the medical field, treatment would be precise, eliminating trial-and-error drug prescription. With a single laboratory test and highly technical computers, a detailed image of the body's system and processes can be automatically spotted including the cause of the disease and its possible treatment. With nanotechnology in the fields of medicine, medical malpractice would be eliminated and the side effects of taking medicines out of sheer guessing from the physicians would be avoided.

11. The drug delivery methods used in chemotherapy has several harmful side effects because of the inaccuracy of drug deliverance at the intended target cell. Researchers at Harvard have been able to attach special RNA strands, measuring about $10 \mathrm{~nm}$ in diameter, to nanoparticles and fill the nanoparticles with a chemotherapy drug. These RNA strands are attracted to cancer cells. When the nanoparticle encounters a cancer cell it adheres to it and releases the drug into the cancer cell. This directed method of drug delivery has great potential for treating cancer patients while producing less side harmful affect than those produced by conventional chemotherapy.

ıı. With highly advanced medical equipment, potential diseases can easily be detected and prevented. Since diseases can be prevented, the quality of life for mankind would be improved and lifespan would be increased.

\subsection{Energy sector:}

The development of more effective energy producing, energy absorbing and storage products in smaller and more efficient devices is possible with this technology. Such items like batteries, solar cells and fuel cells can be made smaller and more effective. 


\subsection{Development of quantum computer:}

Nanotechnology can actually revolutionized a lot of electronic products as nano transistors, nano diodes, quantum computers etc. and their procedures and applications. Quantum computers are groups of billions of customized atoms that are created and manupulated using nanotechnology. Quantum computers represent data differently than any classic computer. While a classical computer represents data in bits that either " 0 " or " 1 ", a quantum computer uses quantum bits to represent " 0 's," "1's,"and both "0's" and "1's" simultaneously. Because quantum bits can represent both 1's and 0's at the same time it is possible to do many calculations simultaneously. A classic computer would do these calculations singularly, making it much slower than a quantum computer. The atoms in a quantum computer function as both a computer's processor and memory. A quantum computer carries out calculations by having the atoms work together in groups of quantum bits, in a highly controlled and isolated environment.

\subsection{Reactivity and strength of materials:}

The nanoparticles are much more reactive than larger particles because their surface area is large as compared to volume. Studies made reveal that nanoparticles of iron can be effective in the cleanup of chemicals in groundwater because they react more efficiently to those chemicals.

As nanosized particles of carbon are extremely strong, bullet proof vest can be made with carbon nanotubes.

\section{ADVERSE EFFECT}

Although nanotechnology has significant impact in all felids of life. It has impressive applications in almost every area of work but it also has some major disadvantages that cannot be neglected. Some of its disadvantages are listed below:

i. One of the biggest disadvantage that world is facing because of nanotechnology is the lack of employment in the fields of traditional farming and manufacturing and industrial sector because of the vast development in the nanotechnology. Nanotech devices and machines have taken place of human to work faster and accurately which has lessen the importance of men power in the field of practical work.

ii. Another big threat, which is born with the advent of nanotechnology, is the easy accessibility of atomic weapons. Nanotechnology has made these weapons more powerful and more destructive. Unauthorized, criminal bodies can reach nuclear weapons easily, and its formulation could be stolen.

iii. Nanotechnology has increased risk to the health also, nanoparticles due to their small size can cause inhalation problem and many other fatal diseases. By just inhaling for 60 seconds in air containing nano particles can damage lungs easily.

iv. At present, nanotechnology is the most expensive technologies and its cost is increasing day by day. The main reason for very high cost is the molecular structure and processing of the product. Huge pricing of nanotech machines make it unaffordable for the common people.

\section{CONCLUSION}

In sum, nanotechnology entails the measurement, prediction and construction of materials on the scale of atoms and molecules. These days, scientists and engineers are taking control of atoms and molecules individually, manipulating them and putting them to use with an extraordinary degree of precision. Though its contributions may seem small at the moment, as funding and manpower continue, this will provide result and innovations. With all the potential that nanotechnology brings, humans must be ready to accept it. We must be ready to properly utilize that which we have discovered. Nanotechnology has the potential to boom and sweep us our feet and with 
so many complex interactions occurring at such a small level, we must be ready to control it. If grows with current rate, nanotechnology will touch the life of nearly every person on the planet in the next few years and it will be the great advancement in earth's history.

\section{REFERENCES}

[1] Charles P. Poole Jr., frank J. Owens, Introduction to Nanotechnology. John Wiley \& sons, Inc.

[2] Paulo Ferreira, Michael F. Ashby, Nanomaterials, Nanotechnologies and Design-An introduction for Engineers and Architects. Butterworth-Heinemann Publication 2009.

[3] Edward L. Wolf, Nanophysics \& Nanotech-
nology-An introduction to modern concepts in Nanoscience.

[4] Sharma P. K., An introduction to nanotechnology and its analysis.

[5] N. Alain, An introduction to nanoscience and nanotechnology.

[6] http://en.wikipedia.org/wiki/implications_of_ nanotechnology.

[7] http://nanogloss.com/nanotechnology/advantages -and-disadvantages of nanotechnology.

[8] http://www.wtec.org/loyola/nano/IWGN.Research.Directions/Chapter01.pdf, 13.

[9] http://www.becon.nih.gov/nstc_def_nano.htm 